\title{
Generalisations of disjunctive sequences
}

\author{
Cristian S. Calude ${ }^{1}$ and Ludwig Staiger*2 \\ ${ }^{1}$ Department of Computer Science, The University of Auckland, \\ Private Bag 92019, Auckland, New Zealand \\ 2 Martin-Luther-Universität Halle-Wittenberg, Institut für Informatik, \\ von-Seckendorff-Platz 1, D-06099 Halle, Germany
}

Received 28 November 2003, revised 7 June 2004, accepted 2 March 2004

Published online 31 January 2005

Key words Disjunctive sequence, Cantor space, Cantor expansion, Baire category, porosity. MSC (2000) 03F60, 68Q45, 54E45

\begin{abstract}
The present paper proposes a generalisation of the notion of disjunctive (or rich) sequence, that is, of an infinite sequence of letters having each finite sequence as a subword. Our aim is to give a reasonable notion of disjunctiveness relative to a given set of sequences $F$. We show that a definition like "every subword which occurs at infinitely many different positions in sequences in $F$ has to occur infinitely often in the sequence" fulfils properties similar to the original unrelativised notion of disjunctiveness. Finally, we investigate our concept of generalised disjunctiveness in spaces of Cantor expansions of reals.
\end{abstract}

(C) 2005 WILEY-VCH Verlag GmbH \& Co. KGaA, Weinheim

\section{Introduction}

A semi-infinite sequence is called disjunctive (or rich) if it has all finite words as subwords (infixes) (cf. [11, 12]). This condition is, obviously, equivalent to having every finite word infinitely often as infix.

The real number associated to a disjunctive sequence $\xi$ over $\{0, \ldots, r-1\}$ is $0 . \xi$. It is interesting to note that in contrast to properties like randomness (cf. [3, 10, 2, 23]) or Kolmogorov complexity (cf. [1, 23]) disjunctiveness is not invariant under base conversion, more precisely speaking, if $\xi \in\{0, \ldots, r-1\}^{\omega}$ and $\eta \in\{0, \ldots, b-1\}^{\omega}$ satisfy $0 . \xi=0 . \eta$ (as reals), then $\xi$ might be disjunctive whereas $\eta$ need not be so. For a more detailed treatment see [9].

Along with the usual base $r$ expansions of real numbers one can also consider so-called Cantor expansions. In general, a Cantor expansion of a real is defined as follows (cf. [6, 8]): Let $f(1), f(2), \ldots, f(n), \ldots$ be a fixed infinite sequence of positive integers greater than 1 and $0 \leq x_{n}<f(n)$, for every $n \geq 1$. The real number

$$
\alpha:=\sum_{i=1}^{\infty} \frac{x_{i}}{f(1) \cdot f(2) \cdots \cdots f(i)}
$$

has $0 . x_{1} x_{2} \ldots$ as (one of) its Cantor expansion(s).

It is easy to see that the set of subwords occurring in a sequence $\xi \in X^{(f)}$, where

$$
X^{(f)}:=\left\{x_{1} x_{2} \ldots x_{i} \ldots: 0 \leq x_{i}<f(i)\right\}
$$

depends on the function $f: \mathbb{N} \longrightarrow \mathbb{N}$. Thus we need a definition of disjunctiveness for Cantor expansions. In this paper we propose a possible modification of the notion of disjunctive sequence in the following way ${ }^{1)}$ : A sequence $\xi \in F \subseteq \mathbb{N}^{\omega}$ is $F$-disjunctive if every infix which occurs at infinitely many different positions in sequences $\eta \in F$ occurs infinitely often in $\xi$. This proposal has some similarity with fairness concepts in which a process is called strongly fair when an action enabled infinitely often is carried out infinitely often (see e.g. [7, 24]). Here, of course, the phrase "which may occur infinitely often in some sequence" needs further specification.

\footnotetext{
* Corresponding author: e-mail: staiger@informatik.uni-halle.de, Phone: +49345 55 24714, Fax: +49345 5527009.

1) We base our generalisation on infinite occurrences of subwords. This proposal seems to be justified by the results of Section 3 .
} 


\section{Preliminaries}

\subsection{Notation}

By $\mathbb{N}=\{0,1,2, \ldots\}$ we denote the set of natural numbers. In order to treat arbitrary finite alphabets we let $X_{r}:=\{0, \ldots, r-1\}$ be an alphabet of cardinality $\left|X_{r}\right|=r, r \in \mathbb{N}, r \geq 2$. In this paper we will use finite alphabets $\left(X_{r}\right)$ and $\mathbb{N}$ as a countably infinite alphabet. In both cases we shall simply write $X$.

By $X^{*}$ we denote the set of finite strings (words) on $X$, including the empty word $e$. We consider also the space $X^{\omega}$ of infinite sequences ( $\omega$-words) over $X$. For $w \in X^{*}$ and $\eta \in X^{*} \cup X^{\omega}$ let $w \cdot \eta$ be their concatenation. This concatenation product extends in an obvious way to subsets $W \subseteq X^{*}$ and $B \subseteq X^{*} \cup X^{\omega}$. If $\left(W_{i}\right)_{i=1}^{\infty}$ is a family of subsets of $X^{*}$, then the infinite product $\prod_{i=1}^{\infty} W_{i}$ is defined as

$$
\prod_{i=1}^{\infty} W_{i}:=\left\{\xi \in X^{\omega}: \xi=w_{1} \cdot w_{2} \cdots w_{i} \cdots, \text { where } w_{i} \in W_{i}\right\} .
$$

If $W_{i}=W$ for all $i$ we will briefly write $W^{\omega}$ instead of $\prod_{i=1}^{\infty} W$.

By “ $\sqsubseteq$ " we denote the prefix relation, that is, $w \sqsubseteq \eta$ if there is an $\eta^{\prime}$ such that $w \cdot \eta^{\prime}=\eta$, and

$$
\operatorname{pref}(\eta):=\left\{w \in X^{*}: w \sqsubseteq \eta\right\} \quad \text { and } \quad \operatorname{pref}(B):=\bigcup_{\eta \in B} \operatorname{pref}(\eta)
$$

are the languages of finite prefixes of $\eta$ and $B$, respectively. The set of subwords (infixes) of $\eta \in X^{*} \cup X^{\omega}$ will be denoted by $\operatorname{infix}(\eta):=\left\{w \in X^{*}: \exists v(v w \sqsubseteq \eta)\right\}$ and $\operatorname{infix}(B):=\bigcup_{\eta \in B} \operatorname{infix}(\eta)$.

In the sequel, we will be mainly interested in sets of the form $X^{(f)}:=\left\{x_{1} x_{2} \ldots: 0 \leq x_{i}<f(i)\right\} \subseteq \mathbb{N}^{\omega}$ which depend on $f: \mathbb{N} \longrightarrow \mathbb{N}$. For the constant function $f(x)=r$ we get the case $X^{(f)}=X_{r}^{\omega}$.

We introduce a metric in $X^{(f)}$ as follows:

$$
\varrho_{f}(\xi, \eta):=\inf \left\{\prod_{i=1}^{|w|} f(i)^{-1}: w \sqsubset \xi \wedge w \sqsubset \eta\right\}
$$

which makes $\left(X^{(f)}, \varrho_{f}\right)$ a compact metric space ${ }^{2)}$.

Theorem 2.1 The metric space $\left(X^{(f)}, \varrho_{f}\right)$ is compact.

It is easily verified that $\varrho_{f}$ is indeed a metric which, in addition, satisfies the ultra-metric inequality:

$$
\varrho_{f}(\zeta, \xi) \leq \max \left\{\varrho_{f}(\zeta, \eta), \varrho_{f}(\xi, \eta)\right\}
$$

Open balls (in view of (3) they are simultaneously closed) in the space $\left(X^{(f)}, \varrho_{f}\right)$ are the sets of the form $X^{(f)} \cap w \cdot \mathbb{N}^{\omega}$. Then open sets in $X^{(f)}$ are of the form $X^{(f)} \cap W \cdot \mathbb{N}^{\omega}$, where $W \subseteq \operatorname{pref}\left(X^{(f)}\right)$. From this it follows that a subset $F \subseteq X^{(f)}$ is closed iff $\operatorname{pref}(\xi) \subseteq \operatorname{pref}(F)$ implies $\xi \in F$. The closure of a subset $F \subseteq X^{(f)}$ in the space $\left(X^{(f)}, \varrho_{f}\right)$, that is, the smallest closed subset of $X^{\omega}$ containing $F$, is denoted by $\mathcal{C}(F)$. One has $\mathcal{C}(F)=\{\xi: \operatorname{pref}(\xi) \subseteq \operatorname{pref}(F)\}$.

It should be mentioned that, due to the special choice of the metric $\varrho_{f}$ (see (2)), the following additivity property for balls is satisfied:

$$
\sum_{x \in X_{f(|w|+1)}} \operatorname{diam}_{f}\left(X^{(f)} \cap w \cdot x \cdot \mathbb{N}^{\omega}\right)=\operatorname{diam}_{f}\left(X^{(f)} \cap w \cdot \mathbb{N}^{\omega}\right),
$$

as $\bigcup_{x \in X_{f(|w|+1)}} X^{(f)} \cap w \cdot x \cdot \mathbb{N}^{\omega}=X^{(f)} \cap w \cdot \mathbb{N}^{\omega}$.

\subsection{Measure}

Using (4) we introduce a measure $\mu$ on $X^{(f)}$ defined on balls as $\mu\left(w \cdot \mathbb{N}^{\omega} \cap X^{(f)}\right):=\operatorname{diam}_{f}\left(w \cdot \mathbb{N}^{\omega} \cap X^{(f)}\right)$ and then extended in the usual way to subsets of $X^{(f)}$ (cf. [16]). This measure has the property that $\mu(F)$ equals the usual Lebesgue measure of the set $\left\{\sum_{i=1}^{\infty} \frac{x_{i}}{f(1) \cdot f(2) \cdots f(i)}: x_{1} x_{2} \ldots x_{i} \cdots \in F\right\} \subseteq[0,1]$.

\footnotetext{
2) There are other possibilities to structure $X^{(f)}$ as a compact metric space. Here we want to stress the similarity between $\left(X^{(f)}, \varrho_{f}\right)$ and the Cantor expansions of real numbers, so we require the property of equation (4) which is implicit in our definition of $\varrho_{f}$.
} 


\subsection{Density and Baire category}

Next we introduce the topological concepts of density and Baire category for our complete metric space $\left(X^{(f)}, \varrho_{f}\right)$ (see e.g. [13, 16]). A subset $F \subseteq X^{(f)}$ is called dense in $X^{(f)}$ provided its closure $\mathcal{C}(F)$ is the whole space $X^{(f)}$. A set $M \subseteq X^{(f)}$ is nowhere dense in $\left(X^{(f)}, \varrho_{f}\right)$ provided its closure $\mathcal{C}(M)$ does not contain a nonempty open subset. A set $F$ is of first Baire category (or meagre) if it is a countable union of nowhere dense sets; otherwise it is of second Baire category. The complements of sets of first Baire category are called residual.

\subsection{Porosity}

A further topic related to density is porosity, considered e. g. in [25, Section 2.C] or [17]. This concept, however, does rely on the particular metric chosen for the space. We explain it for the space $\left(X^{(f)}, \varrho_{f}\right)$. Let $\lambda(E, u):=$ $\sup \left\{\operatorname{diam}_{f}\left(w \cdot \mathbb{N}^{\omega} \cap X^{(f)}\right): u \sqsubseteq w \wedge w \cdot \mathbb{N}^{\omega} \cap E=\emptyset\right\}$ be the diameter of a largest ball contained in $u \cdot \mathbb{N}^{\omega} \cap X^{(f)}$, but disjoint from $E$. The porosity of $E$ at the point $\xi$ is:

$$
\boldsymbol{p}(E, \xi):=\limsup _{u \rightarrow \xi} \frac{\lambda(E, u)}{\operatorname{diam}_{f}\left(u \cdot \mathbb{N}^{\omega} \cap X^{(f)}\right)} .
$$

For example, if $E$ is closed and $\xi \notin E$, then $\boldsymbol{p}(E, \xi)=1$.

A set $E \subseteq X^{(f)}$ is called porous if $\boldsymbol{p}(E, \xi)>0$, for all $\xi \in X^{(f)}$. It is obvious that every porous set is nowhere dense, but the converse need not be true. It should be noted, however, that in $\left(X_{r}^{\omega}, \varrho\right)$ every nowhere dense set definable by a finite automaton is porous (see [18, 21, 22]).

The following connection between porosity and measure in $X^{(f)}$ is immediate by the Lebesgue density theorem ([16, Theorem 3.20]).

Lemma 2.2 Let $E \subseteq X^{(f)}$. If $\mu(E)>0$, then $E$ is not porous in $\left(X^{(f)}, \varrho_{f}\right)$.

\subsection{Disjunctive sequences in $\boldsymbol{X}_{r}^{\boldsymbol{\omega}}$}

Finally, we list some properties of the set of disjunctive sequences $D_{r} \subseteq X_{r}^{\omega}$ known from [4, 22]:

Theorem 2.3

1. $D_{r}=\left\{\xi \in X_{r}^{\omega}:|\operatorname{pref}(\xi) \cap W|=\aleph_{0}\right\}$, where

$$
W:=\left\{w x: w \in X_{r}^{*} \wedge x \in X_{r} \wedge \exists n\left(\operatorname{infix}(w x) \supseteq X_{r}^{n} \wedge \operatorname{infix}(w) \nsupseteq X_{r}^{n}\right)\right\} .
$$

2. $D_{r}$ is $\Pi_{2}$-definable and a residual set in $X_{r}^{\omega}$.

3. $X_{r}^{\omega} \backslash D_{r}=\bigcup_{w \in X_{r}^{*}}\left(X_{r}^{\omega} \backslash X_{r}^{*} \cdot w \cdot X_{r}^{\omega}\right)=\bigcup_{w \in X_{r}^{*}}\left(X_{r}^{|w|} \backslash\{w\}\right)^{\omega}$.

4. $X_{r}^{\omega} \backslash D_{r}$ is the union of all nowhere dense $\omega$-languages definable by a finite automaton.

5. $X_{r}^{\omega} \backslash D_{r}$ is a countable union of porous sets.

6. $\mu\left(D_{r}\right)=1$, for all non-degenerate product measures on $X_{r}^{\omega}$.

\section{Generalised disjunctiveness}

In this section we make precise the fact, stated informally, that an $\omega$-word $\xi \in F$ should be called disjunctive if every word $w \in \mathbb{N}^{*}$ which can appear at infinitely many different positions in sequences in $F$ has to appear infinitely often as an infix of $\xi$.

To this end we observe that a necessary condition for a word $w$ to appear infinitely often as an infix in $F$ is the following one. Let $\operatorname{Infix}_{\infty}(F):=\left\{w: \exists^{\infty} n \exists u(|u|=n \wedge u w \in \operatorname{pref}(F)\}\right.$. An $\omega$-word $\xi$ is called $F$-disjunctive provided $\xi \in F$ and $\operatorname{Infix}_{\infty}(F)=\operatorname{Infix}_{\infty}(\{\xi\}) .{ }^{3)}$

For general subsets of $\mathbb{N}^{\omega}$ or $X^{(f)}$ this condition is complicated. To simplify it we introduce the following notion which, when satisfied for $\operatorname{Infix}_{\infty}(F)$, will alleviate the investigation of disjunctive sequences. A set $W \subseteq \mathbb{N}^{*}$ is referred to as left prolongable if for every $w \in W$ there is an $x \in \mathbb{N}$ such that $x \cdot w \in W$.

\footnotetext{
3) In what follows we shall write $\operatorname{Infix} x_{\infty}(\xi)$ as a shorthand for $\operatorname{Infix}_{\infty}(\{\xi\})$.
} 
Proposition 3.1 Let $\operatorname{Infix}_{\infty}(F)$ be left prolongable. Then, for all $\xi \in F$, $\operatorname{infix}(\xi) \supseteq \operatorname{Infix}_{\infty}(F)$ implies $\operatorname{Infix}_{\infty}(\xi) \supseteq \operatorname{Infix}_{\infty}(F),$.

Proof. Assume $w \in \operatorname{Infix}_{\infty}(F)$ and $w \notin \operatorname{Infix}_{\infty}(\xi)$. Then there exists a longest prefix $u \sqsubset \xi$ such that $u \cdot w \sqsubset \xi$. If $\operatorname{infix}(\xi) \supseteq \operatorname{Infix}_{\infty}(F)$ and $\operatorname{Infix}_{\infty}(F)$ is left prolongable we have a $v,|v|>|u|$, such that $v \cdot w \in \operatorname{infix}(\xi)$, which contradicts the choice of $u$.

The following example shows that prolongability is essential.

Example 3.2 Let $F:=\prod_{i=2}^{\infty}\{i 1,00\}$. Then $\operatorname{Infix}_{\infty}(F)=0^{*} \cup 1 \cdot 0^{*}$, and, indeed, for $\eta=210^{\omega}$ we have $\operatorname{infix}(\eta) \supseteq \operatorname{Infix}_{\infty}(F) \supset \operatorname{Infix}_{\infty}(\eta)=0^{*}$.

As a corollary to Proposition 3.1 we obtain properties of the set of all $F$-disjunctive $\omega$-words similar to those in Theorem 2.3 (for $D_{r}$ ).

Corollary 3.3 If $\operatorname{Infix}_{\infty}(F)$ is left prolongable, then $D_{F}=\bigcap_{w \in \operatorname{Infix}_{\infty}(F)}\left(F \cap \mathbb{N}^{*} \cdot w \cdot \mathbb{N}^{\omega}\right)$ is the set of all F-disjunctive $\omega$-words.

Corollary 3.4 If $\operatorname{Infix}_{\infty}(F)$ is left prolongable, then

$$
F \backslash D_{F}=\bigcup_{w \in \operatorname{Infix}_{\infty}(F)}\left(F \backslash \mathbb{N}^{*} \cdot w \cdot \mathbb{N}^{\omega}\right)=\bigcup_{w \in \operatorname{Infix}_{\infty}(F)}\left(F \cap \bigcap_{j=0}^{|w|-1} \mathbb{N}^{j} \cdot\left(\mathbb{N}^{|w|} \backslash\{w\}\right)^{\omega}\right) .
$$

\section{Disjunctiveness in ultimately connected sets}

Here we consider the case of a subset $F$ of a Cantor space $X_{r}^{\omega}$ having the following property:

$$
\forall u\left(u \in \operatorname{pref}(F) \rightarrow \exists v\left(v \in X_{r}^{*} \wedge u \cdot v \cdot F \subseteq F\right)\right)
$$

These sets, called ultimately connected, can be characterised by the so-called stabiliser of $F \subseteq X_{r}^{\omega}, \operatorname{Stab}(F)$ (cf. $[14,15,19,20])$ :

$$
\operatorname{Stab}(F):=\{w \in \operatorname{pref}(F) \backslash\{e\}: w \cdot F \subseteq F\} .
$$

$\operatorname{Stab}(F)$ is closed under concatenation, so it is a subsemigroup of $X_{r}^{*}$.

Proposition 4.1 An $\omega$-language $F \subseteq X_{r}^{\omega}$ is ultimately connected iff $\operatorname{pref}(F) \subseteq \operatorname{pref}(\operatorname{Stab}(F))$.

Examples of ultimately connected $\omega$-languages are the so-called $\omega$-power languages $W^{\omega}$ when $W \subseteq X_{r}^{*}$. Obviously, the stabiliser of an $\omega$-power language $W^{\omega}$ satisfies

$$
W^{*} \backslash\{e\} \subseteq \operatorname{Stab}\left(W^{\omega}\right) \subseteq \operatorname{Stab}\left(\mathcal{C}\left(W^{\omega}\right)\right) \subseteq \operatorname{pref}\left(W^{\omega}\right) \quad \text { and } \quad \operatorname{Stab}\left(W^{\omega}\right) \cdot W^{\omega}=W^{\omega} .
$$

Here, as usual, we denote the subsemigroup of $X_{r}$ generated by $W$, that is $\bigcup_{i=0}^{\infty} W^{i}$, by $W^{*}$.

For ultimately connected $\omega$-languages $F$ the language $\operatorname{Infix}_{\infty}(F)$ has the following properties.

Proposition 4.2 We have $\operatorname{Infix}_{\infty}(F)=\operatorname{infix}(\operatorname{Stab}(F))$ and $\operatorname{Infix} x_{\infty}(F)$ is both left and right prolongable. Corollary 3.4 applies immediately to ultimately connected $\omega$-languages.

Corollary 4.3 If $F \subseteq X_{r}^{\omega}$ is ultimately connected, then

$$
F \backslash D_{F}=\bigcup_{w \in \operatorname{Stab}(F)}\left(F \backslash X_{r}^{*} \cdot w \cdot X_{r}^{\omega}\right)=\bigcup_{w \in \operatorname{Stab}(F)}\left(F \cap \bigcap_{j=0}^{|w|-1} X_{r}^{j} \cdot\left(X_{r}^{|w|} \backslash\{w\}\right)^{\omega}\right) .
$$

We conclude the part on ultimately connected $\omega$-languages by mentioning some results from [20] similar to Theorem 2.3.4 concerning $\omega$-languages definable by a finite automaton which are nowhere dense in $F$. To this end we mention that a set $E \subseteq X_{r}^{\omega}$ is nowhere dense in $F \subseteq X_{r}^{\omega}$ iff for all $u \in \operatorname{pref}(F)$ there exists $v \in X_{r}^{*}$ such that $u \cdot v \in \operatorname{pref}(F) \backslash \operatorname{pref}(E)$.

Lemma 4.4 If $F \subseteq X_{r}^{\omega}$ is ultimately connected and $E$ is definable by a finite automaton, then we have:

1. $E$ is nowhere dense in $F$ iff there is a $w \in \operatorname{Stab}(F)$ such that $E \cap \mathcal{C}(F) \subseteq \mathcal{C}(F) \backslash \operatorname{Stab}(F)^{*} \cdot w \cdot X_{r}^{\omega}$, and

2. $E \subseteq \mathcal{C}(F)$ is nowhere dense in $F$ iff there is a $\bar{w} \in \operatorname{Stab}(F)$ such that $E \subseteq \mathcal{C}(F) \backslash X_{r}^{*} \cdot \bar{w} \cdot X_{r}^{\omega}$.

A proof can be found in [20, Section 5]. 


\section{Disjunctiveness in $\left(X^{(f)}, \varrho_{f}\right)$}

In this section we derive some simple properties of the set $\operatorname{Infix}_{\infty}\left(X^{(f)}\right)$. From these properties we derive that $\operatorname{Infix}_{\infty}\left(X^{(f)}\right)$ is both left and right prolongable, whence similar properties as those described in Theorem 2.3 and Corollaries 3.3 and 3.4 hold for the set of all disjunctive $\omega$-words in $X^{(f)}, D_{f}$, independently of the choice of $f$.

\subsection{General properties}

We start with a few simple properties.

\section{Proposition 5.1}

1. $u \cdot \mathbb{N}^{\omega} \subseteq \mathbb{N}^{*} \cdot w \cdot \mathbb{N}^{\omega}$ if and only if $w \in \operatorname{infix}(u)$.

2. Let $u \in \operatorname{pref}\left(X^{(f)}\right)$ and $|u| \leq n$. Then $0^{n} \cdot w \in \operatorname{pref}\left(X^{(f)}\right)$ if and only if $u \cdot 0^{n-|u|} \cdot w \in \operatorname{pref}\left(X^{(f)}\right)$.

3. If $w \in \operatorname{Infix}_{\infty}\left(X^{(f)}\right)$, then $\{0,1\}^{*} \cdot w \cdot\{0,1\}^{*} \subseteq \operatorname{Infix}_{\infty}\left(X^{(f)}\right)$.

Proof.

1. The direction from right to left is trivial. Let $a \in\{0,1\}$ be a letter different from the last letter of $w$. Then $u \cdot a^{\omega} \in \mathbb{N}^{\omega}$. If $u \cdot a^{\omega} \in \mathbb{N}^{*} \cdot w \cdot \mathbb{N}^{\omega}$, then $w \in \operatorname{infix}\left(u \cdot a^{\omega}\right)$, and, since $a$ is not the last letter of $w, w \in \operatorname{infix}(u)$ follows.

The other properties are readily seen.

As an immediate consequence of Proposition 5.1.3 we obtain the announced property.

Corollary 5.2 The set $\operatorname{Infix}_{\infty}\left(X^{(f)}\right)$ is left and right prolongable, for every function $f: \mathbb{N} \longrightarrow \mathbb{N} \backslash\{0,1\}$.

Define $\underline{\ell}_{f}(n):=\lim \sup _{i \rightarrow \infty} \min \{f(i+j): 1 \leq j \leq n\}$.

Lemma 5.3 $X_{r}^{n} \subseteq \operatorname{Infix}_{\infty}\left(X^{(f)}\right)$ if and only if $\underline{\ell}_{f}(n) \geq r$.

Proof. We have $\underline{\ell}_{f}(n) \geq r$ iff for every $m \in \mathbb{N}$ there is an $i \geq m$ such that $f(i+j) \geq r$, for $1 \leq j \leq n$. Thus $0^{i} \cdot w \in \operatorname{pref}\left(X^{(f)}\right)$, for every $w \in X_{r}^{n}$.

Conversely, if $X_{r}^{n} \subseteq \operatorname{Infix}_{\infty}\left(X^{(f)}\right)$, then there are infinitely many $i \in \mathbb{N}$ such that $0^{i} \cdot(r-1)^{n} \in \operatorname{pref}\left(X^{(f)}\right)$, whence $f(i+j) \geq r$, for $1 \leq j \leq n$.

The following example shows that $\operatorname{Infix}_{\infty}\left(X^{(f)}\right)$ may indeed not be larger than indicated by Lemma 5.3 and Proposition 5.1.3.

Example 5.4 For the function

$$
f(i):= \begin{cases}i+1 & \text { if } i=n^{2} \\ 2 & \text { otherwise }\end{cases}
$$

we have $\operatorname{Infix}_{\infty}\left(X^{(f)}\right)=\{0,1\}^{*} \cdot \mathbb{N} \cdot\{0,1\}^{*}$.

The contrary might be also true.

Corollary 5.5 If $\lim _{n \rightarrow \infty} f(n)=\infty$, then $\operatorname{Infix}_{\infty}\left(X^{(f)}\right)=\mathbb{N}^{*}$.

\subsection{Computability}

If one considers computable functions $f: \mathbb{N} \longrightarrow \mathbb{N} \backslash\{0,1\}$, then one obtains some computability constraints on $\operatorname{Infix}_{\infty}\left(X^{(f)}\right)$. Using the Tarski-Kuratowski algorithm one can easily deduce an upper bound for the complexity of $\operatorname{Infix}_{\infty}\left(X^{(f)}\right)$ in the arithmetical hierarchy.

Lemma 5.6 If $f$ is a computable function, then $\operatorname{Infix}_{\infty}\left(X^{(f)}\right)$ is $\Pi_{2}$-definable.

The following example shows that we cannot do better even if the function $f$ is bounded. For the case $\lim _{i \rightarrow \infty} f(i)=\infty, \operatorname{Infix}_{\infty}\left(X^{(f)}\right)=\mathbb{N}^{*}$ is computable in view of Corollary 5.2. 
Example 5.7 Let $M \subseteq \mathbb{N}$ be in $\Pi_{2} \backslash \Sigma_{2}$, so it has a representation $M=\left\{n: \exists^{\infty} m((m, n) \in R)\right\}$, where $R \subseteq \mathbb{N} \times \mathbb{N}$ and $R \in \Sigma_{1} \cap \Pi_{1}$. Let $R$ be ordered in some computable way, i. e. $R=g(\mathbb{N})$ for some injective computable function. Then $X^{(f)}:=\prod_{i=0}^{\infty} X_{2}^{g_{1}(i)+1} \cdot X_{4} \cdot X_{3}^{g_{2}(i)} \cdot X_{4}$, where $g(i)$ is the pair $\left(g_{1}(i), g_{2}(i)\right)$. Consequently, $\operatorname{Infix}_{\infty}\left(X^{(f)}\right) \cap 3 \cdot 2^{*} \cdot 3=\left\{3 \cdot 2^{n} \cdot 3: n \in M\right\}$ is in $\Pi_{2} \backslash \Sigma_{2}$, hence $\operatorname{Infix}_{\infty}\left(X^{(f)}\right) \in \Pi_{2} \backslash \Sigma_{2}$.

\section{Topological and metric properties of $X^{(f)} \backslash \mathbb{N}^{*} \cdot w \cdot \mathbb{N}^{\omega}$}

In this section we investigate some topological properties of the set of disjunctive sequences in $X^{(f)}$. First we investigate the relationship to density and measure.

\subsection{Density}

We start with a simple proposition which holds for all functions $f: \mathbb{N} \longrightarrow \mathbb{N} \backslash\{0,1\}$.

Lemma 6.1 The set $X^{(f)} \backslash \mathbb{N}^{*} \cdot w \cdot \mathbb{N}^{\omega}$ is nowhere dense in $\left(X^{(f)}, \varrho_{f}\right)$ whenever $w \in \operatorname{Infix}_{\infty}\left(X^{(f)}\right)$.

Proof. If $w \in \operatorname{Infix}_{\infty}\left(X^{(f)}\right)$, then for every $u \in \operatorname{pref}\left(X^{(f)}\right)$ there is $v \in \mathbb{N}^{*}$ such that $u v w \in \operatorname{pref}\left(X^{(f)}\right)$. Thus $u \cdot \mathbb{N}^{\omega} \cap X^{(f)} \nsubseteq X^{(f)} \backslash \mathbb{N}^{*} \cdot w \cdot \mathbb{N}^{\omega}$, for every $u \in \operatorname{pref}\left(X^{(f)}\right)$.

In contrast to Theorem 2.3.6 the measure property does not hold in general.

Example 6.2 Let $\mu$ be the measure on $X^{(f)}$ introduced in Section 2.2. We consider the following function $f(i):=(i+1)^{2}$ and the set $F:=X^{(f)} \backslash \mathbb{N}^{*} \cdot 0 \cdot \mathbb{N}^{\omega}=\prod_{i=1}^{\infty}\left(X_{f(i)} \backslash\{0\}\right)$. Then, we have

$$
\mu(F)=\prod_{i=1}^{\infty}\left(1-\frac{1}{f(i)}\right)=\prod_{j=2}^{\infty}\left(1-\frac{1}{j^{2}}\right)>0 .
$$

Thus the set $F=X^{(f)} \backslash \mathbb{N}^{*} \cdot 0 \cdot \mathbb{N}^{\omega}$ is nowhere dense but has measure $\mu(F)>0$.

\subsection{Porosity in $X^{(f)}$}

From $[25,17]$ (see also Lemma 2.2) it is known that any porous set $F \subseteq X^{(f)}$ is nowhere dense and has measure $\mu(F)=0$. As we have seen in the preceding section the complement of the set of disjunctive sequences in $X^{(f)}$ may have positive measure. In this section we investigate how this behaviour depends on the function $f$.

A first result and a comparison with Theorem 2.3 show that the case of bounded functions is similar to the case of constant alphabets (see also [5]).

Lemma 6.3 If $f: \mathbb{N} \longrightarrow \mathbb{N}$ is bounded, then for every $w \in \operatorname{Infix}_{\infty}\left(X^{(f)}\right)$ the set $X^{(f)} \backslash \mathbb{N}^{*} \cdot w \cdot \mathbb{N}^{\omega}$ is porous in $X^{(f)}$.

Proof. Let $w \in \operatorname{Infix}_{\infty}\left(X^{(f)}\right)$ and $E:=X^{(f)} \backslash \mathbb{N}^{*} \cdot w \cdot \mathbb{N}^{\omega}$. Then there are infinitely many $n \in \mathbb{N}$ such that $0^{n} \cdot w \in \operatorname{pref}\left(X^{(f)}\right)$. According to Proposition 5.1.2, for every $\xi \in X^{(f)}$ and every prefix $u_{n} \sqsubset \xi$ of length $\left|u_{n}\right|=n$ we have $u_{n} \cdot w \in \operatorname{pref}\left(X^{(f)}\right)$. Then $u_{n} \cdot w \cdot \mathbb{N}^{\omega} \cap X^{(f)}$ is disjoint from $E$, whence $\operatorname{diam}_{f}\left(u_{n} \cdot w \cdot \mathbb{N}^{\omega} \cap X^{(f)}\right) \leq \lambda\left(E, u_{n}\right)$ and

$$
\boldsymbol{p}(E, \xi) \geq \lim \sup _{n \rightarrow \infty} \frac{\operatorname{diam}_{f}\left(u_{n} \cdot w \cdot \mathbb{N}^{\omega} \cap X^{(f)}\right)}{\operatorname{diam}_{f}\left(u_{n} \cdot \mathbb{N}^{\omega} \cap X^{(f)}\right)}=\limsup _{n \rightarrow \infty} \prod_{i=\left|u_{n}\right|+1}^{\left|u_{n}\right|+|w|} \frac{1}{f(i)} \geq b^{-|w|}
$$

for $b:=\max \{f(i): i \in \mathbb{N}\}$.

The case when $f$ is unbounded needs a special treatment. A first result concerns functions $f: \mathbb{N} \longrightarrow \mathbb{N}$ tending to infinity.

Theorem 6.4 Let $f: \mathbb{N} \longrightarrow \mathbb{N} \backslash\{0,1\}$. Then the following properties are equivalent.

1. $\lim _{i \rightarrow \infty} f(i)=\infty$.

2. The set $X^{(f)} \backslash \mathbb{N}^{*} \cdot 0 \cdot \mathbb{N}^{\omega}$ is not porous.

3. For every $w \in \operatorname{Infix}_{\infty}\left(X^{(f)}\right) \backslash\{e\}$ the set $X^{(f)} \backslash \mathbb{N}^{*} \cdot w \cdot \mathbb{N}^{\omega}$ is not porous. 
Pro of. The equivalence of the first and second conditions follows from the fact that

$$
\frac{\operatorname{diam}_{f}\left(u \cdot 0 \cdot \mathbb{N}^{\omega} \cap X^{(f)}\right)}{\operatorname{diam}_{f}\left(u \cdot \mathbb{N}^{\omega} \cap X^{(f)}\right)}=f(|u|+1)^{-1}, \text { for } u \in \operatorname{pref}\left(X^{(f)}\right) .
$$

The other equivalence is a consequence of the inequality

$$
\operatorname{diam}_{f}\left(u \cdot 0 \cdot \mathbb{N}^{\omega} \cap X^{(f)}\right) \geq \operatorname{diam}_{f}\left(u \cdot w \cdot \mathbb{N}^{\omega} \cap X^{(f)}\right),
$$

whenever $u \cdot w \in \operatorname{pref}\left(X^{(f)}\right)$ and $w \neq e$.

For unbounded functions $f: \mathbb{N} \longrightarrow \mathbb{N}$ we introduce the following characteristic number

$$
k_{f}:= \begin{cases}-1 & \text { if all } f^{-1}(k) \text { are finite } \\ \sup \left\{k: f^{-1}(k) \text { is infinite }\right\} & \text { otherwise. }\end{cases}
$$

For technical reasons, we denote by $\hat{u} \cdot \mathbb{N}^{\omega} \cap X^{(f)}$ a ball of largest diameter contained in the ball $u \cdot \mathbb{N}^{\omega} \cap X^{(f)}$ but disjoint from $E$. This condition is equivalent to the fact that $\hat{u}$ is a shortest word having $u$ as prefix and satisfying $\hat{u} \notin \operatorname{pref}(E)$. Then the following holds true.

$$
\frac{\lambda(E, u)}{\operatorname{diam}_{f}\left(u \cdot \mathbb{N}^{\omega} \cap X^{(f)}\right)}=\prod_{i=|u|+1}^{|\hat{u}|} \frac{1}{f(i)} .
$$

We obtain the following sufficient condition for the non-porosity of sets $X^{(f)} \backslash \mathbb{N}^{*} \cdot w \cdot \mathbb{N}^{\omega}$.

Theorem 6.5 If $f: \mathbb{N} \longrightarrow \mathbb{N}$ is unbounded and $k_{f}<\infty$, then for every $i>k_{f}$ and $v, w \in\{0,1\}^{*}$ the set $X^{(f)} \backslash \mathbb{N}^{*} \cdot$ viw $\cdot \mathbb{N}^{\omega}$ is not porous in $X^{(f)}$.

Pro of. First observe that in view of Proposition 5.1.3 the set $\operatorname{Infix}_{\infty}\left(X^{(f)}\right)$ contains the word $v i w \in \mathbb{N}^{*}$. Since $X^{(f)} \backslash \mathbb{N}^{*} \cdot v i w \cdot \mathbb{N}^{\omega} \supseteq X^{(f)} \backslash \mathbb{N}^{*} \cdot i \cdot \mathbb{N}^{\omega}$, it suffices to prove that the latter set is not porous, that is, $\boldsymbol{p}\left(X^{(f)} \backslash \mathbb{N}^{*} \cdot i \cdot \mathbb{N}^{\omega}, \xi\right)=0$, for $\xi \in X^{(f)}$.

Since $k_{f}<\infty$, for every $k>k_{f}$ the set $f^{-1}(k)=\{j: f(j)=k\}$ is finite. Let

$$
\ell_{k}:=1+\sup \left\{j: k_{f}<f(j) \leq k\right\} .
$$

Then $f(j)>k$ whenever $j \geq \ell_{k}$ and $f(j)>k_{f}$.

If $u \in \operatorname{pref}\left(X^{(f)} \backslash \mathbb{N}^{*} \cdot i \cdot \mathbb{N}^{\omega}\right)$ and $|u| \geq \ell_{k}$, then every shortest word $\hat{u} \in \operatorname{pref}\left(X^{(f)}\right)$ with $u \sqsubseteq \hat{u}$ and $\hat{u} \in \mathbb{N}^{*} \cdot i$ has to satisfy $f(|\hat{u}|) \geq i>k_{f}$. Thus $f(|\hat{u}|)>k$ and according to (8),

$$
\frac{\operatorname{diam}_{f}\left(\hat{u} \cdot \mathbb{N}^{\omega} \cap X^{(f)}\right)}{\operatorname{diam}_{f}\left(u \cdot \mathbb{N}^{\omega} \cap X^{(f)}\right)}<\frac{1}{k},
$$

for all $u \sqsubset \xi,|u| \geq \ell_{k}$.

The particular case, when $k_{f}=-1$, that is, when $f$ tends to infinity was treated in Theorem 6.4.

Summarising Lemma 6.3 and Theorems 6.4 and 6.5 we obtain the following exhaustive connection between the porosity of sets of the form $X^{(f)} \backslash \mathbb{N}^{*} \cdot w \cdot \mathbb{N}^{\omega}$ and the behaviour of the function $f$ in case when $k_{f}<\infty$.

Theorem 6.6 Let $f: \mathbb{N} \longrightarrow \mathbb{N}$ be a function such that $k_{f}<\infty$ and let $w \in \operatorname{Infix}_{\infty}\left(X^{(f)}\right) \backslash\{e\}$. Then the following conditions hold:

1. $f$ is bounded iff all sets $X^{(f)} \backslash \mathbb{N}^{*} \cdot w \cdot \mathbb{N}^{\omega}$ are porous.

2. $f$ tends to infinity iff none of the sets $X^{(f)} \backslash \mathbb{N}^{*} \cdot w \cdot \mathbb{N}^{\omega}$ is porous.

3. $f$ is unbounded and $\lim \inf _{i \rightarrow \infty} f(i)<\infty$ iff some of the sets $X^{(f)} \backslash \mathbb{N}^{*} \cdot w \cdot \mathbb{N}^{\omega}$ are porous and some are not.

In the remaining case when $k_{f}=\infty$ (here $f$ is necessarily unbounded and $\liminf _{i \rightarrow \infty} f(i)<\infty$ ) we may have both possibilities not excluded by Theorem 6.4 .

First we give an example showing that every set of the form $X^{(f)} \backslash \mathbb{N}^{*} \cdot w \cdot \mathbb{N}^{\omega}$, where $w \neq e$, is porous. 
Example 6.7 Let $f(n):=2+\left(n-\lfloor\sqrt{n}\rfloor^{2}\right)$. It is well known that $f\left(r^{2}+l\right)=2+l$ whenever $l \leq 2 r$. Thus $f^{-1}(k)$ is infinite for every $k \in \mathbb{N} \backslash\{0,1\}$.

Consider $w \in \mathbb{N}^{*}$. Then $w \in X_{r}^{*}$, for an appropriate $r \in \mathbb{N}$. Choose $u \in \operatorname{pref}\left(X^{(f)}\right)$ with $|u|=s^{2}+r$, where $s \geq r+|w|$. Then $f\left(s^{2}+r+i\right)=2+r+i \geq r$, for $0 \leq i \leq|w|$. Consequently, $u \cdot w \in \operatorname{pref}\left(X^{(f)}\right)$ and we have the inequalities

$$
\frac{\operatorname{diam}_{f}\left(u \cdot w \cdot \mathbb{N}^{\omega} \cap X^{(f)}\right)}{\operatorname{diam}_{f}\left(u \cdot \mathbb{N}^{\omega} \cap X^{(f)}\right)} \geq \prod_{i=s^{2}+r+1}^{s^{2}+r+|w|} \frac{1}{f(i)}=\prod_{i=1}^{|w|} \frac{1}{r+i+2}>0 .
$$

This proves that for $w \in X_{r}^{*}$, the porosity of $X^{(f)} \backslash \mathbb{N}^{*} \cdot w \cdot \mathbb{N}^{\omega}$ is at least $\prod_{i=1}^{|w|}(r+i+2)^{-1}>0$.

The final example covers the case when not all sets of the form $X^{(f)} \backslash \mathbb{N}^{*} \cdot w \cdot \mathbb{N}^{\omega}$ with $w \neq e$ are porous.

Example 6.8 Define

$$
f(n):= \begin{cases}2+\left(n / 5-\lfloor\sqrt{n / 5}\rfloor^{2}\right) & \text { if } n \equiv 0 \quad(\bmod 5) \\ 2 & \text { if } n \equiv \pm 1 \quad(\bmod 5) \\ n & \text { if } n \equiv \pm 2 \quad(\bmod 5) .\end{cases}
$$

Similarly to the previous example, for every $k \in \mathbb{N} \backslash\{0,1\}$ the set $f^{-1}(k)$ is infinite, but for every number $n$ with $n \equiv \pm 1(\bmod 5)$ we have $f(n)=2$. Consider the word 22 . If $u \cdot 22 \in \operatorname{pref}\left(X^{(f)}\right)$, then necessarily $|u|=5 \cdot l+1$, for some $l \in \mathbb{N}$, and, by construction, $0^{5 \cdot l+1} \cdot 22 \in \operatorname{pref}\left(X^{(f)}\right)$, for all $l \in \mathbb{N}$. Thus $22 \in \operatorname{Infix}_{\infty}\left(X^{(f)}\right)$ and if $u \cdot 22 \in \operatorname{pref}\left(X^{(f)}\right)$ we have

$$
\frac{\operatorname{diam}_{f}\left(u \cdot 22 \cdot \mathbb{N}^{\omega} \cap X^{(f)}\right)}{\operatorname{diam}_{f}\left(u \cdot \mathbb{N}^{\omega} \cap X^{(f)}\right)} \leq \frac{1}{f(|u|+1) \cdot f(|u|+2)}=\frac{1}{(|u|+1) \cdot(|u|+2)} .
$$

This shows that the set $X^{(f)} \backslash \mathbb{N}^{*} \cdot 22 \cdot \mathbb{N}^{\omega}$ is not porous in $\left(X^{(f)}, \varrho_{f}\right)$.

Acknowledgements L. Staiger's research was in part supported by Project Number 3602449/9343 of the University of Auckland Research Fund, which is hereby gratefully acknowledged. We are grateful to the anonymous referee for the thorough comments which improved the paper.

\section{References}

[1] J.-Y. Cai and J. Hartmanis, On Hausdorff and topological dimensions of the Kolmogorov complexity of the real line. J. Comput. System Sci. 49, 605 - 619 (1994).

[2] C. S. Calude, Information and Randomness: An Algorithmic Perspective, 2nd Edition (Springer Verlag, Berlin 2002).

[3] C. S. Calude and H. Jürgensen, Randomness as an invariant for number representations. In: Results and Trends in Theoretical Computer Science (H. Maurer, J. Karhumäki, and G. Rozenberg, eds.), Lecture Notes in Computer Science 812, pp. 44 - 66 (Springer-Verlag, Berlin 1994).

[4] C. S. Calude, L. Priese, and L. Staiger, Disjunctive sequences: An overview. CDMTCS Research Report 063, Auckland 1997.

[5] C. S. Calude and T. Zamfirescu, Most numbers obey no probability laws. Publicationes Mathematicae Debrecen 54, Supplement, $619-623$ (1999).

[6] S. Drobot, Real Numbers (Prentice-Hall, Englewood Cliffs, New Jersey, 1964).

[7] N. Francez, Fairness (Springer-Verlag, New York 1986).

[8] G. H. Hardy and E. M. Wright, An Introduction to the Theory of Numbers (Cambridge Univ. Press, London 1954).

[9] P. Hertling, Disjunctive $\omega$-words and real mumbers. J. Universal Computer Science 2, 549 - 568 (1996).

[10] P. Hertling and K. Weihrauch, Random elements in effective topological spaces with measure. Information and Computation 181, $32-56$ (2003).

[11] H. Jürgensen, H. J. Shyr, and G. Thierrin, Disjunctive $\omega$-languages. Elektron. Informationsverarb. Kybernetik EIK 19, $267-278(1983)$

[12] H. Jürgensen and G. Thierrin, On $\omega$-languages whose syntactic monoid is trivial. Intern. J. Comput. Inform Sci. 12 , 359 - 365 (1983)

[13] K. Kuratowski, Topology I (Academic Press, New York 1966).

[14] I. Litovsky, Generateurs des langages rationelles de mots infinis. Thèse, Univ. de Lille Flandres Artois, 1988. 
[15] I. Litovsky, Free submonoids and minimal $\omega$-generators of $R^{\omega}$. Acta Cybernetica $101-2,35-43$ (1991).

[16] J. C. Oxtoby, Measure and Category (Springer-Verlag, Berlin 1971).

[17] D. A. Renfro, Study of Porous and Sigma-Porous Sets (CRC Press 2001).

[18] L. Staiger, Reguläre Nullmengen, Elektron. Informationsverarb. Kybernetik EIK 12, 307 - 311 (1976).

[19] L. Staiger, A note on connected $\omega$-languages, Elektron. Informationsverarb. Kybernetik EIK 16, 245 - 251 (1980).

[20] L. Staiger, On $\omega$-power languages. In: New Trends in Formal Languages, Control, Cooperation, and Combinatorics, (Gh. Păun and A. Salomaa, eds.), Lecture Notes in Computer Science 1218, pp. 377 - 393 (Springer-Verlag, Berlin 1997).

[21] L. Staiger, Rich $\omega$-words and monadic second-order arithmetic. In: Computer Science Logic, 11th International Workshop, CSL'97, Selected Papers (M. Nielsen and W. Thomas, eds.), Lecture Notes in Computer Science 1414, pp. 478 - 490 (Springer-Verlag, Berlin 1998).

[22] L. Staiger, How large is the set of disjunctive sequences?, J. Universal Computer Science 8, 348 - 362 (2002).

[23] L. Staiger, The Kolmogorov complexity of real numbers. Theoret. Comput. Sci. 284, 455 - 466 (2002).

[24] M. Y. Vardi, Verification of concurrent programs: The automata-theoretic framework. Annals Pure and Appl. Logic 51, $79-98$ (1991).

[25] L. Zajíček, Porosity and $\sigma$-porosity. Real Analysis Exch. 13, 314 - 350 (1987/88). 